\title{
Clinical versus laboratory detection of alcohol abuse: the alcohol clinical index
}

\author{
H A SKINNER, S HOLT, W J SHEU, Y ISRAEL
}

\begin{abstract}
To determine reliable indicators of alcohol abuse a comprehensive set of clinical and laboratory information was acquired from three groups of subjects with a wide range of drinking histories: 131 outpatients with alcohol problems, 131 social drinkers, and 52 patients from family practice. Findings from clinical examination provided greater diagnostic accuracy than laboratory tests for detecting alcohol abuse. Logistic regression analysis produced an overall accuracy of $85-91 \%$ for clinical signs, $84-88 \%$ for items from the medical history, and $71-83 \%$ for laboratory tests in differentiating the three groups. Further analyses showed 17 clinical signs and 13 medical history items that formed a highly diagnostic instrument (alcohol clinical index) that could be used in clinical practice. A probability of alcohol abuse exceeding $\mathbf{0 . 9 0}$ was found if four or more clinical signs or four or more medical history items from the index were present. Despite recent emphasis on the laboratory diagnosis of alcohol abuse simple clinical measures seem to provide better diagnostic accuracy.
\end{abstract}

\section{Introduction}

Although many patients are excessive drinkers, their alcohol problems often pass unrecognised.$^{1.3}$ Problem drinkers consult their doctors at a higher rate than average and tend to use diagnostic facilities more often than the general population. ${ }^{45}$ The failure to identify alcohol problems may be partly due to the doctor's diagnostic approach. In clinical practice doctors tend to concentrate on physical sequelae of alcohol abuse at the expense of recording a drinking history and relevant psychosocial information. ${ }^{6}$ This may impede detecting the young problem drinker, who generally has fewer laboratory or clinical signs of diseases associated with alcohol abuse but often exhibits social and psychological problems related to excessive drinking. ${ }^{70}$

The assessment of psychosocial factors using interview and questionnaire methods is of major value in detecting alcohol abuse. ${ }^{10}$ Biomedical research has also indicated the usefulness of various laboratory tests, such as $\gamma$-glutamyltransferase activity and mean corpuscular volume, as markers of excessive drinking. ${ }^{11-13}$ Several recent studies have underscored the diagnostic advantage of combining biomedical and psychosocial data. ${ }^{14-16}$ Laboratory tests and clinical signs possess more objectivity for diagnosis than self reports of alcohol problems, which may be subject to denial. Laboratory tests, however, possess only moderate sensitivity for detecting alcohol abuse. ${ }^{1314^{17}}$ Furthermore, the stratification of clinical signs

Addiction Research Foundation, Toronto, Canada M5S 2S1

H A SKINNER, PHD, senior scientist and professor, department of preventive medicine and biostatistics, University of Toronto, Canada

W J SHEU, PHD, statistician

Y ISRAEL, PHD, head of biochemical research and professor, departments of medicine and pharmacology, University of Toronto, Canada

Division of Gastroenterology, Southern Illinois University School of Medicine, Springfield, Illinois, USA

S HOLT, MB, FACP, chief

Correspondence and requests for reprints to: Dr Skinner. of alcohol abuse in terms of their diagnostic importance has received little consideration. ${ }^{\prime \prime}$

Published reports continue to highlight selected findings on clinical examination that may be related to excessive drinking. ${ }^{11} 18$ The possibility that clinical or laboratory indicators may be insensitive or redundant for the detection of alcohol abuse has not been explored in sufficient detail. ${ }^{19}$ We therefore studied a comprehensive set of 108 potential indicators of alcohol abuse to establish a short list of clinical and laboratory criteria for identifying alcohol abuse.

\section{Methods}

We compiled 108 clinical and laboratory tests from an extensive review of relevant studies ${ }^{1011}$ and used them to examine 314 subjects, who comprised three groups with a wide range of drinking histories (outpatients with alcohol problems, social drinkers, and family practice patients). To evaluate the discriminating power of different tests under ideal conditions 131 outpatients with documented alcohol problems were compared with 131 social drinkers. These groups were matched for age (mean age 38 (11 SD) years) and sex ( 86 men, 45 women). The outpatients with a broad range of alcohol problems were voluntarily seeking help from the Addiction Research Foundation, Toronto. Their mean daily alcohol intake in the previous six months had been $130(120.8 \mathrm{SD}) \mathrm{g}$ ethanol. They had been excessive drinkers - that is, consuming more than $400 \mathrm{~g}$ ethanol/week-for a mean of $7 \cdot 7(6 \cdot 7)$ years and reported a broad range of consequences related to drinking as assessed by the Michigan alcoholism screening test ${ }^{20}:$ mean score $26 \cdot 3(9 \cdot 4)$, where a score of five or more suggested problem drinking. The social drinkers from the Toronto area consumed fewer than seven drinks ( $95 \mathrm{~g}$ ethanol) per week and no more than two drinks ( $27 \mathrm{~g}$ ethanol) per occasion and had low scores on the Michigan alcoholism screening test: mean $0 \cdot 3(2 \cdot 3)$.

A group of 61 patients from family practice (Toronto area) were used to make the study results more applicable to routine medical practice. Nine subjects were excluded because they reported excessive drinking (more than $60 \mathrm{~g}$ on average per day). This was done to provide a comparison group of patients who were not excessive drinkers. There were no differences $(\mathrm{p}>0.28)$ in age and sex between the 52 family practice patients and the other two groups (mean age $37(15) ; 29$ men, 23 women). The daily intake of alcohol by this group was $13 \cdot 8(15 \cdot 1) \mathrm{g}$ ethanol, and their mean score on the Michigan alcoholism screening test was low at $1 \cdot 4(3 \cdot 9)$.

Each subject underwent a standardised physical examination that focused on signs associated with excessive drinking. " The four doctors conducting the examination were not directly associated with the project, although they were aware of the drinking status of subjects. The 42 clinical signs were elicited in a systematic format and grouped according to the organ or system affected (table I). A self administered medical history questionnaire was developed to include a comprehensive assessment of symptoms associated with alcohol abuse. ${ }^{11}$ The 44 items were grouped in table II according to nine clinical domains, and their empirical clustering was verified using item analysis procedures. A comprehensive range of 22 laboratory tests (table III) was performed on a sample of venous blood. The alcohol markers section in table III included tests that are commonly related to excessive drinking. ${ }^{13-15}$ Other tests in table III were grouped according to haematology, urea, and electrolytes (sequential multiple analysis 6), or routine chemistry (sequential multiple analysis 12) for comparison with previous investigations of these series. ${ }^{12}$ The Michigan alcoholism screening test was administered and alcohol intake was estimated by a standardised, self administered questionnaire. The drinking history was corroborated by a detailed interview in which lifetime alcohol use was documented. ${ }^{21}$

Clinical and laboratory items were compared between groups using either a $\chi^{2}$ test for qualitative variables or a $t$ test for quantitative variables. Because of the large number of variables evaluated (42 clinical signs, 22 laboratory tests, 44 medical history items) a stringent measure of significance, p $>0.001$, was used when interpreting univariate statistical tests in tables I, II, and III. 
The diagnostic value of composite indices of clinical measures was investigated using logistic regression analysis from the biomedical computer programs $P$ series statistical software package. ${ }^{22}$ This multivariate procedure estimates the probability of alcohol abuse occurring, conditional on a linear function of clinical measures, through the logistic distribution functional form:

$$
\text { probability of alcohol abuse }=\frac{\mathrm{e}^{a+\beta i x}}{1+\mathrm{e}^{\mathrm{a}+\beta_{\mathrm{x}}}}
$$

where e (approximately $2 \cdot 718$ ) is the base of the natural logarithms, $\alpha$ is a constant, and $\beta \mathrm{x}$ is the weighted linear combination of measures-for example, neurological signs. A cutpoint on the probabilities was chosen that scores for medical history items were used as input for logistic regression analyses. A predictor variable was considered useful in table $\mathrm{V}$ if its logistic regression coefficient divided by its standard error exceeded $2 \cdot 0$.

\section{Results and comment}

The prevalence of physical signs associated with alcohol abuse is given in table I. A broader range of clinical findings was noted in the outpatients with alcohol problems than in either the social drinkers or family practice patients. With respect to vital signs, a significant difference $(p<0.001)$ was

TABLE I-Clinical signs

\begin{tabular}{|c|c|c|c|}
\hline & $\begin{array}{l}\text { Social drinkers } \\
\quad(n=131)\end{array}$ & $\begin{array}{l}\text { Alcohol outpatients } \\
\qquad(\mathrm{n}=131)\end{array}$ & $\begin{array}{l}\text { Family practice patients } \\
\qquad(\mathrm{n}=52)\end{array}$ \\
\hline & & $\operatorname{Mean}(S D)$ & \\
\hline \multicolumn{4}{|l|}{ Vital signs: } \\
\hline Peak expiratory flow rate $(1 / \mathrm{min})$ & $440 \cdot 0(124 \cdot 9)$ & $348 \cdot 5(115 \cdot 7)$ & Not measured \\
\hline Systolic blood pressure ( $\mathrm{mm} \mathrm{Hg}$ ) & $127 \cdot 9(17 \cdot 3)$ & $130 \cdot 7(18 \cdot 7)$ & $123.4(14.5)$ \\
\hline Diastolic blood pressure $(\mathrm{mm} \mathrm{Hg})$ & $77 \cdot 4(13.6)$ & $82.0(13.5)$ & $74.8(10.6)$ \\
\hline Pulse rate (beats $/ \mathrm{min}$ ) & $73 \cdot 6(12 \cdot 3)$ & $76 \cdot 3(10 \cdot 9)$ & $73 \cdot 5(6 \cdot 5)$ \\
\hline Body mass: weight $(\mathrm{g}) /$ height $^{2}(\mathrm{~cm})$ & $2.49(0.4)$ & $2 \cdot 40(0 \cdot 4)$ & $2 \cdot 49(0 \cdot 4)$ \\
\hline \multicolumn{4}{|l|}{ Neurological signs: } \\
\hline${ }^{\star}$ Hand tremort & $1 \cdot 3(0 \cdot 6)$ & $1.8(0 \cdot 7)$ & $1 \cdot 0(0 \cdot 0)$ \\
\hline$\star^{\star}$ Tandem gaitf & $1 \cdot 1(0 \cdot 3)$ & $1.4(0.7)$ & $1 \cdot 0(0 \cdot 1)$ \\
\hline *Deep knee bend $t$ & $1.3(0.6)$ & $1.7(0.7)$ & $1.0(0.3)$ \\
\hline Reflexes (biceps, knees, ankles) $\ddagger$ & $1.5(0.4)$ & $1.6(0.3)$ & $1 \cdot 0(0 \cdot 1)$ \\
\hline Muscle wasting (arms, legs) $\dagger$ & $1 \cdot 0(0 \cdot 1)$ & $1 \cdot 1(0 \cdot 4)$ & $1 \cdot 0(0.0)$ \\
\hline Limb tendernesst & $1 \cdot 0(0 \cdot 2)$ & $1 \cdot 0(0 \cdot 2)$ & $1 \cdot 0(0 \cdot 1)$ \\
\hline Glove and stocking anaesthesia $\dagger$ & $1 \cdot 0(0 \cdot 2)$ & $1 \cdot 1(0 \cdot 3)$ & $1.0(0.0)$ \\
\hline \multicolumn{4}{|l|}{ Liver and abdomen signs: } \\
\hline$\star$ Spider naevi $(>5)$ & 4 & 42 & 3 \\
\hline *Collateral circulation & $1 \cdot 1(0 \cdot 3)$ & $1.4(0.5)$ & $1 \cdot 0(0 \cdot 0)$ \\
\hline Liver tendernesst & $1 \cdot 0(0 \cdot 1)$ & $1.2(0.5)$ & $1 \cdot 1(0 \cdot 2)$ \\
\hline${ }^{\star}$ Gynaecomastia & $7 \%$ & $29 \%$ & $2 \%$ \\
\hline${ }^{\star} \mathrm{Abdomen}$ tenderness $\dagger$ & $1 \cdot 1(0 \cdot 3)$ & $1 \cdot 3(0 \cdot 4)$ & $1 \cdot 1(0 \cdot 3)$ \\
\hline Liver enlargement $\int$ & $0 \cdot 2(0 \cdot 8)$ & $1 \cdot 8(2 \cdot 8)$ & $2 \cdot 7(4 \cdot 9)$ \\
\hline Spleen enlargement $\delta$ & $0.0(0 \cdot 2)$ & $0 \cdot 1(0 \cdot 6)$ & $0.3(1 \cdot 7)$ \\
\hline Peripheral oedemat & $1 \cdot 0(0 \cdot 0)$ & $1.04(0 \cdot 2)$ & $1.06(0.3)$ \\
\hline \multirow[t]{2}{*}{ Ascitest } & $1 \cdot 0(0 \cdot 0)$ & $1 \cdot 05(0 \cdot 2)$ & $1.0(0.0)$ \\
\hline & \multicolumn{3}{|c|}{ No (\%) present } \\
\hline Face inspection: & & & \\
\hline${ }^{\star}$ Rhinophyma & $7(5)$ & $31(24)$ & $1(2)$ \\
\hline *Facial erythema & $17(13)$ & $46(35)$ & $3(6)$ \\
\hline Periorbital oedema & $1(1)$ & $12(9)$ & $1(2)$ \\
\hline Engorged vessels in conjunctivae & $24(18)$ & $38(29)$ & $2(4)$ \\
\hline Arcus senilis & $16(12)$ & $20(15)$ & 0 \\
\hline \multicolumn{4}{|l|}{ Oral inspection: } \\
\hline${ }^{\star}$ Coated tongue & $9(7)$ & $63(48)$ & $3(6)$ \\
\hline$\star$ Oedema of soft palate & $24(18)$ & $71(54)$ & $3(6)$ \\
\hline Poor oral hygiene & $7(5)$ & $30(23)$ & $4(7)$ \\
\hline Parotid swelling & $5(4)$ & $20(15)$ & $2(4)$ \\
\hline Fetor of alcohol & $1(1)$ & $8(6)$ & $3(6)$ \\
\hline \multicolumn{4}{|l|}{ Hand inspection: } \\
\hline${ }^{\star}$ Nicotine stains & $4(3)$ & $72(55)$ & $5(10)$ \\
\hline ^Palmar erythema & $10(8)$ & $37(28)$ & $3(6)$ \\
\hline Bitten nails & $20(15)$ & $31(24)$ & $6(11)$ \\
\hline White nails & $29(22)$ & $42(32)$ & $2(4)$ \\
\hline Finger clubbing & $5(4)$ & $12(9)$ & $1(2)$ \\
\hline Dupuytren's contracture & $3(2)$ & $1(1)$ & $1(2)$ \\
\hline \multicolumn{4}{|l|}{ Bruises, scars, and burns: } \\
\hline ^Bruises/abrasions & $12(9)$ & $69(53)$ & $3(6)$ \\
\hline$\star$ Scars (secondary to trauma) & $41(31)$ & $96(73)$ & $10(19)$ \\
\hline${ }^{\star}$ Cigarette burns & 0 & $35(27)$ & $1(2)$ \\
\hline ^Tattoos & $7(5)$ & $35(27)$ & $4(7)$ \\
\hline Seborrhoea/dermatitis & $35(27)$ & $29(22)$ & $1(2)$ \\
\hline
\end{tabular}

p $<0.001$ for distinguishing social drinkers from alcohol outpatients, as well as alcohol outpatients from family practice patients $\left(t\right.$ test or $\left.\chi^{2}\right)$.

tGraded $(1=$ normal, $2=$ mild, $3=$ moderate, $4=$ severe $)$.

fGraded $(1=$ normal, 2 =abnormal); index score is the average for left and right biceps, knees, and ankles.

Measured in cm below costal margin.

maximised the accuracy of classification for distinguishing patients with alcohol problems from those without (social drinkers or family practice patients). Diagnostic sensitivity, specificity, and predictive power were determined according to standard procedures (see table IV). ${ }^{23}$

A separate logistic regression analysis was conducted within each group of laboratory tests in table III to allow comparisons with previous studies that have examined these tests. The clinical signs and medical history items were grouped by clinical domains (tables I and II), and each group was scored by adding individual results. Selected groups of clinical signs (vital signs, neurological signs, and liver or abdomen signs) were converted into normal deviate scores (mean $0, S D 1$ ) before addition in order to put them into the same metric. The seven summary scores for clinical signs and nine summary evident between the peak expiratory flow rate of social drinkers and that of outpatients with alcohol problems, and the outpatients had a higher diastolic blood pressure than either the social drinkers or family practice patients $(\mathrm{p}<0.01)$. Several neurological findings (hand tremor, tandem gait, and deep knee bend) consistently differentiated the outpatients from either social drinkers or family practice patients. Signs of liver disease were also important diagnostic factors (table I). Inspection of the face, mouth, and hands showed several items that clearly distinguished outpatients with alcohol problems from the other two groups. Also clinical signs of past or present injury (bruises, scars, or burns) were common among those who abused alcohol.

The outpatients with alcohol problems had many varied symptoms 
associated with alcohol abuse (table II). More than half of the symptoms listed in table II separated the outpatients with alcohol problems from both the social drinkers and family practice patients; alcohol withdrawal symptoms, anxiety, and depression in particular. The laboratory test results are given in table III. Both $\gamma$-glutamyltransferase and mean corpuscular volume significantly differentiated $(\mathrm{p}<0.001)$ the outpatients with alcohol problems from the social drinkers and family practice patients. On routine blood chemistry, albumin and aspartate aminotransferase activity differentiated excessive from normal drinkers, and the outpatients had a lower mean blood urea nitrogen value than the other subjects. signs achieved an overall accuracy of $91 \%$ for diagnosis, with good sensitivity $(93 \%)$ and positive predictive value $(95 \%)$. The most useful diagnostic findings on examination included neurological signs, face and mouth inspection, signs of injury, and liver-abdomen signs. The medical history and routine chemistry had good diagnostic sensitivity $(90 \%$ and $93 \%$, respectively). Lower specificity was expected because of the greater likelihood of abnormal clinical findings unrelated to excessive drinking among the family practice patients.

The most diagnostic clinical signs and items from the medical history are listed in table VI. This brief list was compiled by identifying which clinical

TABLE II-Medical history items. Values are numbers (and percentages) positive for each item

\begin{tabular}{|c|c|c|c|}
\hline & $\begin{array}{l}\text { Social drinkers } \\
\quad(n=131)\end{array}$ & $\begin{array}{l}\text { Alcohol outpatients } \\
\qquad(\mathrm{n}=131)\end{array}$ & $\begin{array}{l}\text { Family practice patients } \\
\qquad(\mathrm{n}=52)\end{array}$ \\
\hline \multicolumn{4}{|l|}{ Trauma history (since 18th birthday): } \\
\hline${ }^{\star}$ Injured in an assault or fight & $8(6)$ & $64(49)$ & $7(13)$ \\
\hline Injured in a road traffic accident & $17(13)$ & $41(31)$ & $10(19)$ \\
\hline Head injury & $22(17)$ & $67(51)$ & $14(26)$ \\
\hline *Injury after drinking & $4(3)$ & $75(57)$ & $7(13)$ \\
\hline Fractures or dislocations & $29(32)$ & $71(54)$ & $17(32)$ \\
\hline \multicolumn{4}{|l|}{ Anxiety or depression: } \\
\hline${ }^{\star}$ Often anxious & $39(30)$ & $88(67)$ & $19(37)$ \\
\hline${ }^{\star}$ Often depressed & $18(14)$ & $81(62)$ & $14(27)$ \\
\hline Often has temper outbursts & $16(12)$ & $55(42)$ & $16(29)$ \\
\hline${ }^{\star}$ Recurrent insomnia & $17(13)$ & $73(56)$ & $5(10)$ \\
\hline${ }^{\star}$ Contemplated suicide & $52(4)$ & $45(34)$ & $5(9)$ \\
\hline${ }^{\star}$ Attempted suicide & $3(2)$ & $31(24)$ & $2(4)$ \\
\hline \multicolumn{4}{|l|}{ Gastrointestinal symptoms (frequent): } \\
\hline Heartburn-indigestion & $21(16)$ & $45(34)$ & $10(19)$ \\
\hline Difficulty in swallowing & $3(2)$ & $20(15)$ & $1(2)$ \\
\hline Regurgitation & $4(3)$ & $13(10)$ & $3(6)$ \\
\hline Acid & $8(6)$ & $30(23)$ & $8(15)$ \\
\hline Diarrhoea & $7(5)$ & $38(29)$ & $5(10)$ \\
\hline$\star$ Wakes up feeling thirsty & $25(19)$ & $86(66)$ & $16(31)$ \\
\hline Unpleasant taste in mouth & $33(25)$ & $88(67)$ & $24(47)$ \\
\hline$\star$ Dry coated tongue & $9(7)$ & $56(43)$ & $8(15)$ \\
\hline * Vomits from drinking & $14(11)$ & $92(70)$ & $13(25)$ \\
\hline \multicolumn{4}{|l|}{ Morning symptoms (often): } \\
\hline Retches or vomits & $1(1)$ & $22(17)$ & $1(2)$ \\
\hline ^Feels sick & $3(2)$ & $35(27)$ & 1 (2) \\
\hline Does not eat breakfast & $31(24)$ & $69(53)$ & $31(60)$ \\
\hline${ }^{\star}$ Loses appetite & $10(8)$ & $63(48)$ & $8(16)$ \\
\hline Feels tired & $39(30)$ & $86(66)$ & $21(40)$ \\
\hline \multicolumn{4}{|l|}{ Psychoperceptual withdrawal: } \\
\hline${ }^{\star}$ Hallucinations & $10(8)$ & $56(43)$ & $3(6)$ \\
\hline ॠMemory loss after drinking & $13(10)$ & $11(84)$ & $7(13)$ \\
\hline$\star$ Blackouts after drinking & $24(18)$ & $12(93)$ & $14(26)$ \\
\hline *Unable to concentrate & $18(14)$ & $71(54)$ & $10(19)$ \\
\hline${ }^{\star}$ Mental confusion & $9(7)$ & $58(44)$ & $12(9)$ \\
\hline${ }^{\star} \mathrm{Bad}$ at remembering recent events & $7(5)$ & $54(41)$ & $5(4)$ \\
\hline \multicolumn{4}{|l|}{$\begin{array}{l}\text { Psychophysical withdrawal: } \\
\text { Pal }\end{array}$} \\
\hline${ }^{\star}$ Delirium tremens & 0 & $42(32)$ & 0 \\
\hline${ }^{\star}$ Hands shake in morning & $1(1)$ & $42(32)$ & $1(2)$ \\
\hline ^Troubled by frightening dreams & $7(5)$ & $45(34)$ & $6(12)$ \\
\hline${ }^{\star}$ Headaches & $8(6)$ & $38(29)$ & $4(8)$ \\
\hline Feels cold or shivers & $12(9)$ & $42(32)$ & $10(19)$ \\
\hline${ }^{\star}$ Hands often tremble & $3(2)$ & $48(37)$ & 0 \\
\hline \multicolumn{4}{|l|}{ Neuropathy symptoms (one month or more): } \\
\hline Numbness or tingling in legs & $7(5)$ & $14(11)$ & $1(2)$ \\
\hline Notable loss of strength in arms or legs & $5(4)$ & $18(14)$ & $5(10)$ \\
\hline \multicolumn{4}{|l|}{ Sexual problems: } \\
\hline ^Experienced sexual problems & $10(8)$ & $37(28)$ & $4(8)$ \\
\hline Loss of sexual drive & $21(16)$ & $34(26)$ & $7(13)$ \\
\hline Has had venereal disease & $13(10)$ & $28(21)$ & $4(8)$ \\
\hline \multicolumn{4}{|l|}{ Respiratory symptoms (three months): } \\
\hline${ }^{\star}$ Coughs most days & $12(9)$ & $50(38)$ & $5(10)$ \\
\hline${ }^{\star}$ Brings up phlegm & $12(9)$ & $55(42)$ & $7(14)$ \\
\hline
\end{tabular}

test or $\left.\chi^{2}\right)$.

Table $\mathrm{V}$ summarises results from the logistic regression analyses. In comparing outpatients and social drinkers, we found that the highest overall accuracy of $88 \%$ was achieved using items in the patient's history. The clinical domains that were most powerful discriminators included: psychoperceptual withdrawal, psychophysical withdrawal, indicators of trauma, and gastrointestinal complaints (table V). Selected clinical signs also provided useful diagnostic information with an overall accuracy of $85 \%$. These signs included inspection of hands and mouth, signs of past or recent injuries, and findings on abdominal examination. Laboratory tests were less powerful than the medical history and clinical signs in distinguishing the outpatients with alcohol problems from social drinkers $(71-76 \%$ overall accuracy). The alcohol laboratory markers had good specificity $(90 \%)$ for ruling out cases, but relatively lower sensitivity $(71 \%)$ for detecting alcohol abuse.

Table $\mathrm{V}$ also summarises the results of comparing alcohol outpatients and family practice patients on the clinical and laboratory measures. The clinical domains were the most powerful discriminators in the logistic regression analyses. Subsequently, items were selected within these clinical domains that significantly differentiated $(p<0.001)$ alcohol outpatients from both social drinkers and family practice patients (tables I and II). The likelihood that a given number of positive findings would be expected in heavy drinkers was computed, based on the odds ratio. ${ }^{24}$ The probability of alcohol abuse in figures 1 and 2 rose steeply with an increase in abnormal clinical findings. With a cut off point of four or more clinical signs (fig 1) or four or more medical history items (fig 2) the probability of alcohol abuse exceeded 0.90 .

Further results on diagnostic accuracy are given in table VII. The clinical signs and medical history items had positive predictive values exceeding $90 \%$, which supported their use for diagnosis since few false positive decisions were being made. A low false positive rate is important in clinical practice to avoid errors in identifying normal drinkers as alcohol abusers. These clinical items, however, had lower negative predictive values, which meant that certain cases of alcohol abuse would have been undetected (false 
negatives). When the clinical signs and medical history items were combined in the alcohol clinical index (table VII) this diagnostic rule improved sensitivity $(89 \%)$ and negative predictive value $(88-92 \%)$ and maintained high specificity $(88-92 \%)$ and positive predictive value $(88-97 \%)$. Overall $88-90 \%$ accuracy was achieved by this simple diagnostic rule (clinical signs four or more or medical history four or more).

\section{Discussion}

In recent years considerable efforts have been directed at the evaluation of laboratory tests as indicators of excessive drinking and alcohol abuse ${ }^{25}$ at the expense of considering clinical information. ${ }^{1011}$ Laboratory tests, such as $\gamma$-glutamyltransferase and mean corpuscular volume, tend to have specificity for ruling out cases but only moderate sensitivity $(30 \%$ to $40 \%)$ for detecting cases of alcohol abuse in ambulatory populations. Various attempts have been made to improve diagnostic accuracy by combining laboratory tests to form an index. Ryback et al have found that a composite index based on commonly ordered laboratory tests (haematology, sequential multiple analysis 6 , sequential multiple analysis 12 series) can increase diagnostic accuracy. ${ }^{12}$ Other investigations have improved diagnostic accuracy by combining laboratory tests and

TABLE III-Laboratory test results. Values are means (SD in parentheses)

\begin{tabular}{|c|c|c|c|}
\hline Test and reference ranges & $\begin{array}{l}\text { Social drinkers } \\
\quad(n=131)\end{array}$ & $\begin{array}{l}\text { Alcohol outpatients } \\
\qquad(n=131)\end{array}$ & $\begin{array}{l}\text { Family practice patients } \\
\qquad(\mathrm{n}=52)\end{array}$ \\
\hline \multicolumn{4}{|l|}{ Alcohol markers: } \\
\hline Blood alcohol concentration $(\mathrm{mmol} / \mathrm{l})$ & 0 & $9 \cdot 4(45 \cdot 7)$ & 0 \\
\hline${ }^{*} \gamma$-Glutamyltransferase $(11-51 \mathrm{U} / 1 \mathrm{men})(7-33 \mathrm{U} / 1$ women $)$ & $22 \cdot 0(20 \cdot 0)$ & $49 \cdot 7(66 \cdot 6)$ & $24 \cdot 7(16 \cdot 0)$ \\
\hline High density lipoprotein $(0.8-1.8 \mathrm{mmo} / \mathrm{l})$ & $1 \cdot 4(0 \cdot 3)$ & $1.5(0.3)$ & $1 \cdot 4(0 \cdot 3)$ \\
\hline${ }^{\star}$ Mean corpuscular volume $(76-96 \mathrm{f})$ & $88 \cdot 5(7 \cdot 3)$ & $95 \cdot 2(6 \cdot 8)$ & $90 \cdot 7(4 \cdot 5)$ \\
\hline \multicolumn{4}{|l|}{ Haematology (including mean corpuscular volume): } \\
\hline Red blood cell count $\left(4 \cdot 0-6 \cdot 2 \times 10^{12} / 1\right.$ men $)\left(4 \cdot 0-5 \cdot 5 \times 10^{12} / 1\right.$ women $)$ & $4 \cdot 8(0 \cdot 5)$ & $4 \cdot 8(0 \cdot 8)$ & $4 \cdot 8(0.4)$ \\
\hline White blood cell count $\left(5 \cdot 0-10.0 \times 10^{9} / 1\right)$ & $6 \cdot 2(1 \cdot 7)$ & $7 \cdot 2(1 \cdot 8)$ & $6.8(2 \cdot 0)$ \\
\hline Haemoglobin concentration $(130-180 \mathrm{~g} / 1 \mathrm{men})(120-160 \mathrm{~g} / \mathrm{l}$ women $)$ & $144 \cdot 1(12 \cdot 9)$ & $149 \cdot 2(17 \cdot 4)$ & $146 \cdot 9(15 \cdot 2)$ \\
\hline Packed cell volume $(0.40-0.541 \mathrm{men})(0.37-0.471$ women $)$ & $0.43(0.07)$ & $0.45(0.04)$ & $0.44(0.04)$ \\
\hline Platelet count $\left(150-500 \times 10^{9} / 1\right)$ & $249 \cdot 3(59 \cdot 0)$ & $239 \cdot 8(66 \cdot 7)$ & $252 \cdot 3(84 \cdot 2)$ \\
\hline \multicolumn{4}{|l|}{ Urea and electrolytes: } \\
\hline ^Urea nitrogen $(3 \cdot 0-6 \cdot 4 \mathrm{mmol} / \mathrm{l})$ & $5 \cdot 6(1 \cdot 6)$ & $14 \cdot 3(1 \cdot 3)$ & $5 \cdot 3(1 \cdot 6)$ \\
\hline Chloride $(98-109 \mathrm{mmol} / \mathrm{h})$ & $103 \cdot 2(2 \cdot 6)$ & $102 \cdot 3(2 \cdot 9)$ & $102 \cdot 4(3 \cdot 2)$ \\
\hline Carbon dioxide (24-32 mmol/ $)$ & $27 \cdot 8(2 \cdot 3)$ & $27 \cdot 9(2 \cdot 1)$ & $28 \cdot 5(2 \cdot 4)$ \\
\hline Potassium $(3.5 .5 .0 \mathrm{mmol} / \mathrm{l})$ & $4 \cdot 4(3 \cdot 3)$ & $4 \cdot 4(0 \cdot 4)$ & $4.5(0.4)$ \\
\hline Sodium $(135-150 \mathrm{mmol} / \mathrm{l})$ & $140 \cdot 1(1 \cdot 8)$ & $139 \cdot 7(2 \cdot 1)$ & $140 \cdot 1(2 \cdot 3)$ \\
\hline \multicolumn{4}{|l|}{ Routine chemistry } \\
\hline Alkaline phosphatase (56-244 U/l) & $164 \cdot 2(48 \cdot 8)$ & $206 \cdot 2(64 \cdot 4)$ & $180 \cdot 7(46 \cdot 6)$ \\
\hline Total protein $(64-80 \mathrm{~g} / \mathrm{l})$ & $73(5 \cdot 0)$ & $75(5 \cdot 0)$ & $74(4 \cdot 0)$ \\
\hline${ }^{\star A}$ Albumin $(40-50 \mathrm{~g} / \mathrm{l})$ & $46(4 \cdot 0)$ & $48(3.0)$ & $46(3 \cdot 0)$ \\
\hline Total bilirubin $(2-18 \mu \mathrm{mol} / \mathrm{l})$ & $8 \cdot 5(6 \cdot 8)$ & $6.8(3 \cdot 4)$ & $8 \cdot 5(3 \cdot 4)$ \\
\hline ^Aspartate aminotransferase (8-30 U/1) & $21.0(7.9)$ & $32 \cdot 5(38 \cdot 0)$ & $20 \cdot 7(6 \cdot 5)$ \\
\hline Alanine aminotransferase $(6-30 \mathrm{U} / \mathrm{l})$ & $18 \cdot 3(8 \cdot 7)$ & $33 \cdot 0(48 \cdot 9)$ & $20 \cdot 1(8 \cdot 6)$ \\
\hline Urate $(172-494 \mu \mathrm{mo} / \mathrm{l})$ & $303(83)$ & $309(83)$ & $321(89)$ \\
\hline Cholesterol $(3 \cdot 6-7 \cdot 0 \mathrm{mmol} / \mathrm{l})$ & $5 \cdot 4(1 \cdot 0)$ & $6 \cdot 0(1 \cdot 1)$ & $5 \cdot 2(1 \cdot 0)$ \\
\hline
\end{tabular}

${ }^{\star} \mathrm{p}<0.001$ ( $t$ test) for distinguishing social drinkers from alcohol outpatients, as well as alcohol outpatients from family practice patients.

Conversion: SI to traditional units -Urea nitrogen: $1 \mathrm{mmol} /=2 \cdot 75 \mathrm{mg} / 100 \mathrm{ml}$. Urate: $1 \mathrm{mmol} / 1=16.81 \mathrm{mg} / 100 \mathrm{ml}$. Cholesterol: $1 \mathrm{mmol} / \mathrm{l}=38.67 \mathrm{mg} / 100$ $\mathrm{ml}$. Bilirubin: $1 \mu \mathrm{mol} / \mathrm{l}=0.06 \mathrm{mg} / 100 \mathrm{ml}$

TABLE IV-Formulas for sensitivity and specificity

\begin{tabular}{lcc}
\hline & \multicolumn{2}{c}{ Group } \\
\cline { 2 - 3 } & Alcohol outpatient & Normal drinker \\
\hline Predicted result: & & \\
Alcohol abuse & $\mathrm{a}$ & $\mathrm{b}$ \\
Normal & $\mathrm{c}$ & $\mathrm{d}$
\end{tabular}

Sensitivity $=a /(a+c)$, specificity $=d /(b+d)$, positive predictive value $=a /(a+b)$, negative predictive value $=d /(c+d)$, overall accuracy $=(a+d) /(a+b+c+d)$ questionnaire data. ${ }^{1416}$ For example, an index based on three laboratory tests $(\gamma$-glutamyl-transferase, mean corpuscular volume, and high density lipoprotein) and a brief history of trauma achieved a diagnostic sensitivity exceeding $80 \%$ while maintaining $90 \%$ specificity. ${ }^{14}$

There is a paucity of research that has attempted to use simple clinical information for detecting alcohol abuse. ${ }^{78}$ Our data from clinical examination provided better diagnostic accuracy than laboratory tests (table V), which are invasive and provide delayed information. We found a set of clinical signs and items in the

TABLE V—Diagnostic value of clinical and laboratory data using logistic regression analysis

\begin{tabular}{|c|c|c|c|c|c|}
\hline & $\begin{array}{l}\text { Sensitivity } \\
\%\end{array}$ & $\begin{array}{l}\text { Specificity } \\
\%\end{array}$ & $\begin{array}{c}\text { Positive predictive value } \\
\%\end{array}$ & $\begin{array}{c}\text { Negative predictive value } \\
\%\end{array}$ & $\begin{array}{c}\text { Overall accuracy } \\
\%\end{array}$ \\
\hline \multicolumn{6}{|l|}{ Distinguishing alcohol outpatients from social drinkers: } \\
\hline Clinical signs & 73 & 94 & 91 & 81 & 85 \\
\hline Medical history & 92 & 84 & 85 & 91 & 88 \\
\hline \multicolumn{6}{|l|}{ Laboratory tests: } \\
\hline Haematology & 84 & 71 & 78 & 77 & 78 \\
\hline Urea and electrolytes & 68 & 74 & 73 & 71 & 72 \\
\hline Routine chemistry & 82 & 70 & 71 & 79 & 76 \\
\hline Alcohol markers: $\gamma$-glutamyltransferase, mean corpuscular volume, high density & & & & & \\
\hline lipoprotein, blood alcohol concentration & 71 & 90 & 79 & 89 & 71 \\
\hline \multicolumn{6}{|l|}{ Distinguishing alcohol outpatients from family practice patients: } \\
\hline Clinical signs & 93 & 87 & 95 & 83 & 91 \\
\hline Medical history & 90 & 69 & 88 & 73 & 84 \\
\hline \multicolumn{6}{|l|}{ Laboratory tests: } \\
\hline Haematology & 92 & 37 & 76 & 69 & 74 \\
\hline Urea and electrolytes & 98 & 16 & 74 & 80 & 74 \\
\hline Routine chemistry & 93 & 58 & 84 & 78 & 83 \\
\hline \multicolumn{6}{|l|}{ Alcohol markers: $\gamma$-glutamyltransferase, mean corpuscular volume, high density } \\
\hline lipoprotein, blood alcohol concentration & 84 & 50 & 76 & 63 & 72 \\
\hline
\end{tabular}


TABLE VI-Alcohol clinical index

\begin{tabular}{|c|c|}
\hline Clinical signs ${ }^{\star}$ & Medical history items \\
\hline $\begin{array}{l}\text { Hand tremor } \\
\text { Tandem gait } \\
\text { Deep knee bend } \\
\text { Spider naevi }(>5) \\
\text { Collateral circulation } \\
\text { Gynaecomastia } \\
\text { Abdominal tenderness } \\
\text { Rhinophyma } \\
\text { Facial erythema } \\
\text { Coated tongue } \\
\text { Oedema of soft palate } \\
\text { Nicotine stains } \\
\text { Palmar erythema } \\
\text { Bruises or abrasions } \\
\text { Scars (secondary to trauma) } \\
\text { Cigarette burns } \\
\text { Tattoos }\end{array}$ & $\begin{array}{l}\text { Inability to concentrate } \\
\text { Troubled by mental confusion } \\
\text { Difficulty in remembering recent events } \\
\text { Hallucinations } \\
\text { Hands shake in morning } \\
\text { Troubled by frightening dreams } \\
\text { Wake up with a headache } \\
\text { Hands often tremble } \\
\text { Injured in an assault or fight } \\
\text { Wake up feeling thirsty } \\
\text { Dry coated tongue } \\
\text { Cough on most days } \\
\text { Bring up phlegm }\end{array}$ \\
\hline
\end{tabular}

^Definitions and instructions for using the alcohol clinical index may be obtained by writing to the first author (HAS).
Alcoholism is impractical for routine use in clinical practice, ${ }^{18}$ and our study reinforces a previous finding that many of the council's criteria are redundant for the diagnosis of alcohol abuse. ${ }^{19} \mathrm{~A}$ clinical examination based on the Le Go grid has been widely used in France for the early detection and medical diagnosis of alcoholism. ${ }^{26}$ Only limited data, however, are available on the diagnostic validity of the Le Go method. ${ }^{27}$ The combination of psychosocial and medical data can improve diagnostic accuracy over use of questionnaire methods alone. ${ }^{78}$ Our study indicates that certain clinical findings can be used to complement psychosocial or laboratory data for improving the identification of alcohol abuse.

Further study of the alcohol clinical index is encouraged in different populations and clinical settings. (A form giving details of how to administer the index is available from HAS.) Our outpatients with alcohol problems averaged under 40 years of age (roughly two thirds between 27 and 49) and had a wide range of drinking histories and alcohol related problems. With older patients a reduction in diagnostic specificity might be observed because of

TABLE VII-Diagnostic value of alcohol clinical index

\begin{tabular}{|c|c|c|c|c|c|}
\hline & $\begin{array}{c}\text { Sensitivity } \\
\%\end{array}$ & $\underset{\%}{\text { Specificity }}$ & $\begin{array}{c}\text { Positive predictive value } \\
\%\end{array}$ & $\begin{array}{c}\text { Negative predictive value } \\
\%\end{array}$ & $\begin{array}{c}\text { Overall accuracy } \\
\%\end{array}$ \\
\hline \multicolumn{6}{|c|}{ Distinguishing alcohol outpatients from social drinkers: } \\
\hline Clinical signs (cutpoint $\geqslant 4$ ) & 75 & 93 & 92 & 79 & 84 \\
\hline Medical history (cutpoint $\geqslant 4$ ) & 69 & 94 & 92 & 75 & 82 \\
\hline Clinical signs $\geqslant 4$ or medical history $\geqslant 4$ & 89 & 88 & 88 & 88 & 88 \\
\hline \multicolumn{6}{|c|}{ Distinguishing alcohol outpatients from family practice patients: } \\
\hline Clinical signs (cutpoint $\geqslant 4$ ) & 75 & 99 & 99 & 61 & 81 \\
\hline Medical history (cutpoint $\geqslant 4$ ) & 69 & 94 & 97 & 56 & 77 \\
\hline Clinical signs $\geqslant 4$ or medical history $\geqslant 4$ & 89 & 92 & 97 & 92 & 90 \\
\hline
\end{tabular}

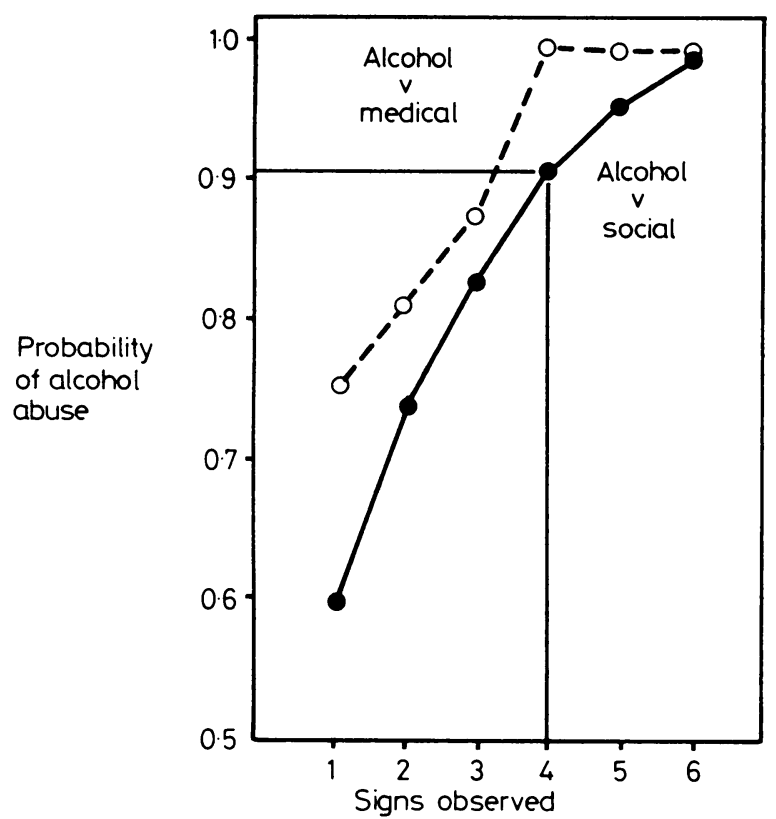

FIG 1-Probability of alcohol abuse estimated at different cutpoints of clinical signs observed on the alcohol clinical index. Outpatients with alcohol problems were compared with social drinkers and with family practice patients.

medical history (table VI) that could discriminate the outpatients with alcohol problems from social drinkers and family practice patients. This brief alcohol clinical index yields information that is highly diagnostic of alcohol abuse (table VI). These findings underscore the diagnostic value of taking a careful medical history and eliciting a short list of clinical signs.

Several attempts have been made to standardise methods for the diagnosis of alcoholism using physiological and clinical data. The extensive list of criteria prepared by the National Council on

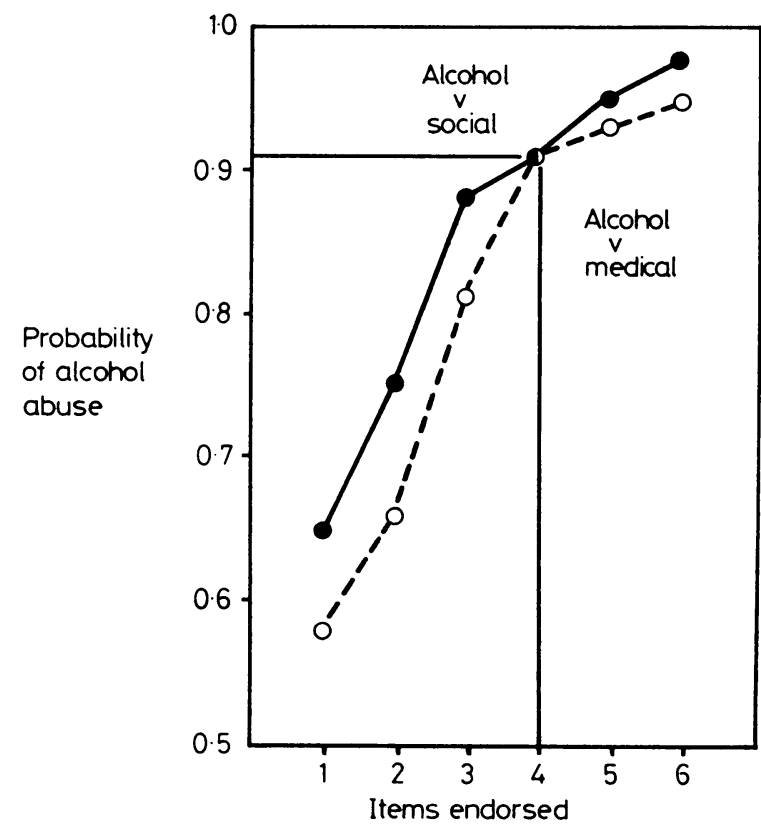

FI, 2-Probability of alcohol abuse estimated at different cutpoints of medical history items endorsed on the alcohol clinical index.

the increased likelihood of some abnormal clinical findings unrelated to alcohol abuse. Accordingly, higher cut off points than four clinical signs or four medical history items may be necessary in an older population for obtaining reasonable diagnostic accuracy. With a predominately younger population-for example, early $20 \mathrm{~s}$ - that has fewer abnormal clinical findings, ${ }^{7.9}$ lower cut off scores on the alcohol clinical index might be necessary for maintaining diagnostic sensitivity. The present study used matched (by age and sex) samples of ambulatory subjects who were representative of social drinkers as well as outpatients in alcohol 
treatment clinics and in general practices. Additional investigations of the alcohol clinical index are planned in other contexts, such as specialist medical wards (for example, gastroenterology and psychiatry) and emergency service departments, where one might expect a higher prevalence of excessive drinking and alcohol related morbidity among patients.

The present study has provided a comprehensive comparison of the relative diagnostic value of clinical and laboratory information for the identification of alcohol abuse. Our findings underscore the value of selected items from the medical history and clinical signs, which can be combined to form an objective index. Previously, incomplete knowledge of the diagnostic power of specific clinical items has prevented firm recommendations about indicators of excessive drinking. " Our advice is to administer the alcohol clinical index routinely during clinical examination and to corroborate it by a brief questionnaire on alcohol problems such as the Michigan alcoholism screening test or CAGE (cut down on drinking, annoyed by others criticizing your drinking, guilt feelings over drinking, eyeopener or drink in the morning), ${ }^{15228}$ as well as by laboratory tests determining mean corpuscular volume and $\gamma$-glutamyltransferase activity. ${ }^{14}$ This practical strategy could make significant inroads on identifying drinking problems that often remain undetected in medical wards and general practices.

\section{References}

1 Wilkins RH. The hidden alcoholic in general practice: a method of detection using a questionnaire. London: Clerk, 1974.

2 McIntosh ID. Alcohol-related disabilities in general hospital patients: a critical assessment of the evidence. Int I Addict 1982;17:609-39.

3 Holt S, Stewart IC, Dixon JM, Elton RA, Taylor TV, Little K. Alcohol and the emergency service Holt S, Stewart IC, Dixon JM, Elton RA,

4 Buchan IC, Buckley EH, Deacon GLS, etal. Problem drinkers and their problems. $7 R$ Coll Gen Pract 1981;31:151-3.

5 Kristensson $\mathrm{H}$, Lunden $\mathrm{A}$, Nitsson BE. Fracture incidence and diagnostic roentgen in alcoholics. Acta Orthop Scand 1980;51:205-7.
6 Jankowski CB, Drum DE. Criteria for the diagnosis of alcoholism. Arch Intern Med 1977;137 1532-6.

7 Skinner HA, Holt S, Allen BA, Haakonson NH. Correlation between medical and behavioral data in the assessment of alcoholism. Alcoholism 1980;4:371-7.

8 Fisher JC, Mason RL, Fisher JV. A diagnostic formula for alcoholism. I Stud Alcohol 1976;37:1247-55.

9 Lee K, Moller L, Hardt F, Haubek A, Jensen E. Alcohol-induced brain damage and liver damage in young males. Lancet 1979;ii:759-61.

10 Skinner HA, Holt $\mathrm{S}$, Israel $Y$. Early identification of alcohol abuse: critical issues and psychosocial indicators for a composite index. Can Med Assoc $\mathcal{J} 1981 ; 124: 1141-52$.
int

11 Holt S, Skinner HA, Israel Y. Early identification of alcohol abuse: clinical and laboratory indicators. Can Med Assoc J 1981;124:1279-94.

12 Ryback RS, Echardt MJ, Felsher B, Rawlings RR. Biochemical and hematologic correlates of alcoholism and liver diseases. JAMA 1982;248:2261-5.

13 Chick J, Kreitman N, Plant $M$. Mean cell volume and gamma-glutamyl transpeptidase as marker of drinking in working men. Lancet $1981 ; \mathrm{i}: 1249-51$.

14 Skinner HA, Holt S, Schuller R, Roy J, Israel Y. Identification of alcohol abuse using laboratory tests and a history of trauma. Ann Intern Med 1984;101:847-51.

5 Bernadt MW, Mumford J, Taylor C, Smith B, Murray RM. Comparison of questionnaire and laboratory tests in the detection of excessive drinking and alcoholism. Lancet 1982;i:325-8.

16 Bernadt MW, Mumford J, Murray RM. A discriminant-function analysis of screening tests for excessive drinking and alcoholism. I Stud Alcohol 1984;45:81-6.

7 Cushman P, Jacobson G, Barboriak JJ, Anderson AJ. Biochemical markers for alcoholism: sensitivity problems. Alcoholism (NY) 1984:8.253-7.

18 National Council on Alcoholism. Criteria for the diagnosis of alcoholism. Ann Intern Med 1972;77:249-58.

19 Ringer $\mathrm{C}$, Kufner H, Antons K, Feuerlein W. The NCA criteria for the diagnosis of alcoholism. Stud Alcohol 1977;38:1254-73.

20 Selzer ML. The Michigan alcoholism screening test: the quest for a new diagnostic instrument. Am f Psychiatry 1971;127:165-8.

21 Skinner HA, Sheu WJ Reliability of alcohol use indices: the lifetime drinking history and the MAST. I Stud Alcohol 1982;43:1157-70.

22 Dixon WJ, ed. Biomedical computer programs. Berkeley: University of California Press, 1981.

23 Sox HC. Probability theory in the use of diagnostic tests. Ann Intern Med 1986;104:60-6.

24 Fleiss JL. Statistical methods for rates and proportions. New York: Wiley, 1981.

25 Johnson RD, Williams R. Prevention of hazardous drinking: the value of laboratory tests. Br Med I 1985;290:1849-50.

26 Le Go PM. A propos de la lutte contre l'alcoolisme dans une grande collectivite de travail. Bull Acad Natl Med 1967;151:197-205.

27 Babor TF, Weill J, Treffardier M, Bernard JY. Detection and diagnosis of alcohol dependence using the Le Go grid method. In: Chang NC, Chao HM, eds. Early identification of alcohol abuse. Rockville, Maryland: National Institute on Alcohol Abuse and Alcoholism, 1985. (Research Rockville, Maryland: National Institure on Alcohol Abuse and Alcoholism, 1985. (Research
monograph 17. Skinner

Allen BA, McIntosh MC, Palmer WH. Lifestyle assessment: just asking makes difference. Br Med J 1985;290:214-6.

\begin{abstract}
The amount of starch escaping absorption in the small intestine was measured in eight patients with symptomatic diverticular disease and eight controls. Unabsorbed starch was calculated from breath hydrogen measurements after a potato meal compared with the hydrogen response to lactulose. The proportion of unabsorbed starch was low in all the patients (mean 3.3\%) and was only about a quarter of that in the controls $(12.4 \% ; \mathrm{p}<0.01)$.

These findings confirm that unabsorbed starch provides an important quantity of carbohydrate reaching the colon and suggest that super efficient starch absorption, by reducing this provision, may promote the development of diverticular disease.

Department of Medicine, St James's University Hospital, Leeds LS9 7TF J R THORNTON, MD, MRCP, tutor in medicine, honorary senior registrar A DRYDEN, BSC, SRD, dietitian

J KELLEHER, PHD, senior lecturer in biochemistry in relation to medicine M S LOSOWSKY, MD, FRCP, professor of medicine

Correspondence to: Dr Thornton.
\end{abstract}

\section{Introduction}

Recent work has shown that dietary starch is not completely absorbed by the small intestine. ${ }^{12}$ The degree of malabsorption of starch varies with different food sources. ${ }^{13}$ Furthermore, one recent study found wide variation in starch malabsorption among people eating the same meal, with between $2 \%$ and $20 \%$ of the starch escaping absorption in the small intestine.

There is considerable evidence that a low intake of dietary fibre predisposes to diverticular disease ${ }^{+6}$ Fibre is the main exogenous source of colonic carbohydrate. In view of the possibility that unabsorbed starch may also contribute a sizable quantity" of carbohydrate to the colon we hypothesised that people who absorb starch particularly well, such that little reaches their colon, might be more at risk of diverticular disease. We call this better than average starch absorption "super efficient."

\section{Subjects and methods}

We studied eight patients with symptomatic diverticular disease. They had presented complaining of lower abdominal pain, with or without altered bowel habit, and had at least 10 colonic diverticula seen on barium enema. 